\title{
The emerging role of myeloid-derived suppressor cells in lung diseases
}

\author{
Saeed Kolahian ${ }^{1,2}$, Hasan Halit Öz ${ }^{1}$, Benyuan Zhou ${ }^{1}$, Christoph M. Griessinger ${ }^{3}$, \\ Nikolaus Rieber ${ }^{1,4}$ and Dominik Hartl ${ }^{1}$ \\ Affiliations: \\ ${ }^{1}$ Children's Hospital of the University of Tübingen, Pediatric Infectiology, Immunology \& Cystic Fibrosis, \\ Tübingen, Germany. \\ ${ }^{2}$ Dept of Basic Science, Faculty of Veterinary Medicine, University of Tabriz, Tabriz, Iran. \\ ${ }^{3}$ Werner Siemens Imaging Center, Dept of Preclinical Imaging and Radiopharmacy, Eberhard Karls University \\ Tübingen, Tübingen, Germany. \\ ${ }^{4}$ Dept of Pediatrics, Kinderklinik München Schwabing, Klinikum rechts der Isar, Technische Universität \\ München, Munich Germany.
}

\section{Correspondence:}

Dominik Hartl, Children's Hospital of the University of Tübingen, Pediatric Infectiology, Immunology \& Cystic Fibrosis, Herrenbergerstrasse 59/1, 72076 Tübingen, Germany.

E-mail: Dominik.hartlamed.uni-tuebingen.de

ABSTRACT Myeloid-derived suppressor cells (MDSCs) are innate immune cells characterised by their potential to control T-cell responses and to dampen inflammation. While the role of MDSCs in cancer has been studied in depth, our understanding of their relevance for infectious and inflammatory disease conditions has just begun to evolve. Recent studies highlight an emerging and complex role for MDSCs in pulmonary diseases. In this review, we discuss the potential contribution of MDSCs as biomarkers and therapeutic targets in lung diseases, particularly lung cancer, tuberculosis, chronic obstructive pulmonary disease, asthma and cystic fibrosis.

@ERSpublications

Myeloid-derived suppressor cells are involved in various lung diseases and represent promising therapeutic targets http://ow.ly/WKZKh

Received: Sept 222015 | Accepted after revision: Dec 152015 | First published online: Feb 042016

Support statement: D. Hartl was supported by the German Research Foundation (Deutsche Forschungsgemeinschaft, Emmy Noether Programme) grant number HA 5274/3-1 and CRC/SFB685 S. Kolahian was supported by the Humboldt Foundation. Funding information for this article has been deposited with FundRef.

Conflict of interest: None declared.

Copyright OERS 2016 


\section{Myeloid-derived suppressor cells \\ Definition}

Suppressive myeloid cells were initially identified more than three decades ago in patients with cancer [1-3]. Later on, these cells were uniformly termed as myeloid-derived suppressor cells (MDSCs) [4] and defined by 1) their myeloid origin and 2) their ability to suppress T-cell responses. Despite a plethora of studies on MDSCs in mice and men, the precise haematopoietic origin and lineage-association still remain a matter of debate $[5,6]$. Broadly accepted is the notion that MDSCs stem from immature myeloid cells (IMCs) and can be subdivided into granulocytic/neutrophilic MDSCs (G-MDSCs) and monocytic MDSCs (M-MDSCs). Current MDSC concepts suggest that differentiation of IMCs into mature granulocytes, macrophages or dendritic cells (DCs) in bone marrow is skewed towards MDSCs in cancers [7]. While tumour-derived factors, such as granulocyte/macrophage colony-stimulating factor (GM-CSF), have been proposed to induce MDSCs in malignancies, the signals that drive MDSC generation in non-malignant infectious and inflammatory conditions remain incompletely understood $[7,8]$. MDSCs are not defined as a single subset of cells, but rather represent a group of phenotypically heterogeneous myeloid cells that share a common biological activity. Human MDSCs have been described to commonly express the myeloid markers CD11b and CD33 as well as CD66b/CD15 for G-MDSCs and CD14 for M-MDSCs [9]. Murine MDSCs express the surface markers $\mathrm{CD} 11 \mathrm{~b}$ and Gr1 and lack the expression of cell-surface markers that are specific for macrophages or DCs [10]. Sub-phenotyping divides murine MDSCs into monocytic (Ly-6G ${ }^{\text {low }} \mathrm{CD} 11 \mathrm{~b}^{+}$Ly-6C ${ }^{\text {high }} \mathrm{SSC}^{\text {low }}$ ) and granulocytic/neutrophilic (Ly-6G ${ }^{\text {high }} \mathrm{CD} 11 b^{+}$Ly-6C $\left.{ }^{\text {intermed }}\right)$ subsets $[7,11]$.

\section{Expansion}

MDSCs have been reported to expand in malignant, infectious and autoimmune conditions $[7,8]$. The factors driving MDSC expansion comprise a broad variety of pro-inflammatory factors, including interferon (IFN) $\gamma$ [12-14], cyclooxygenase (COX) 2, stem-cell factor and prostaglandin E (PGE) $[15,16]$, GM-CSF [17], transforming growth factor (TGF) $\beta$ [18-21], and interleukin (IL) 1 $\beta$ [22]. Signal transducer and activator of transcription 3 (STAT3) is regarded as the main transcription factor that regulates the expansion of MDSCs. STAT3 activation increases survival and proliferation of myeloid progenitor cells and regulates MDSC expansion through inducing the expression of S100A8 and S100A9 proteins $[23,24]$. The combination of IL-6 and GM-CSF has been identified to generate MDSCs from both human and murine immune cells $[25,26]$. Beyond GM-CSF and IL-6, microbial factors have been described to induce MDSCs. Particularly, the opportunistic bacterium Pseudomonas aeruginosa was found to potently induce MDSC generation through flagellin [27]. Moreover, fungal infections with Aspergillus fumigatus and Candida albicans induced a distinct subset of MDSCs through the pattern recognition receptor Dectin-1 and its downstream adaptor protein caspase recruitment domain-containing protein 9, which further involves the generation of reactive oxygen species (ROS) as well as caspase- 8 activity and IL- 1 production [28]. The retinoblastoma gene was found to regulate M-MDSC differentiation towards G-MDSCs in tumour-bearing mice [29]. Under hypoxic conditions in the tumour microenvironment, MDSCs suppress both antigen-specific and non-specific T-cell activity via hypoxia-inducible factor (HIF) $1 \alpha$ [30]. HIF- $1 \alpha$ also redirects MDSCs differentiation toward tumour-associated macrophages, which further supports the immune-suppressive network in the tumour microenvironment [30]. MDSCs have been reported to have a shorter lifespan in comparison to their counterparts, granulocytes and monocytes, in tumour-free mice mediated by tumour necrosis factor (TNF)-related apoptosis-induced ligand receptors and endoplasmic reticulum stress [31]. LPS and IFN- $\gamma$ treatment in combination has been shown to trigger the expansion of splenic myeloid precursors into functionally suppressive MDSCs, blocking their development into DCs [32]. In addition, it was reported that G-MDSCs are expanded in neonatal cord blood and efficiently modulate innate and adaptive immune responses by suppressing $\mathrm{T}$ and natural killer (NK) cell responses [33]. These neonatal cord blood MDSCs may weaken cellular anti-microbial host defence responses and may contribute to the increased lung infection susceptibility in neonates.

\section{Function}

The mechanisms listed below have been implicated in MDSC-mediated suppression of T-cell function:

\section{Reactive oxygen species}

ROS have been implicated in MDSC-derived T-cell suppression as common mechanism in neoplastic conditions, inflammation and microbial infections [34]. MDSCs in both tumour-bearing mice and patients with cancer produce ROS and inhibition of ROS production diminished the suppressive effect of MDSCs [35-38]. It has been further shown that ligation of integrins, expressed on MDSCs, contribute to increased ROS production following the interaction of MDSCs with T-cells [39]. Several cytokines, such as TGF- $\beta$, IL-6, and GM-CSF, have been described to induce the production of ROS by MDSCs [40]. 
Arginase, nitric oxide synthase and nitric oxide

Arginase (ARG) 1 and inducible nitric oxide synthase (iNOS) are involved in L-arginine metabolism. iNOS generates nitric oxide (NO) from L-arginine, and arginase converts L-arginine into urea and L-ornithine. MDSCs express high levels of arginase and iNOS, and utilise these enzymes to deprive arginine and, thereby, inhibit T-cell function [41-43]. The shortage of L-arginine inhibits T-cell proliferation by decreasing T-cellular $\mathrm{CD} 3 \zeta$ expression [44] and inhibiting the upregulation of the cell-cycle regulators cyclin D3 and cyclin-dependent kinase 4 [45]. On the other hand, NO suppresses T-cell function through inhibition of Janus kinase 3 and STAT5 in T-cells [46], inhibition of MHC class II expression [47] and the induction of T-cell apoptosis [48].

\section{Peroxynitrite}

Peroxynitrite is one of the most powerful oxidants, and is a product of a chemical reaction between NO and superoxide anion $\left(\mathrm{O}_{2}^{-}\right)$. Peroxynitrite induces the nitration and nitrosylation of the amino acids cysteine, methionine, tryptophan and tyrosine. Increased levels of peroxynitrite are associated with tumour progression in many types of cancer [49-54], which has been linked with T-cell unresponsiveness.

Induction of regulatory $T$-cells

MDSCs were found to promote the development of $\mathrm{CD} 4{ }^{+} \mathrm{CD} 25^{+} \mathrm{FOXP} 3^{+}$regulatory T-cells (Tregs), an effect that required the activation of tumour-specific T-cells and the presence of IFN- $\gamma$ and IL-10 $[55,56]$.

Subset-specific mechanisms

The two main subsets of MDSC employ different mechanisms to suppress T-cell proliferation. The G-MDSC expresses high levels of ROS and low levels of NO, whereas the M-MDSC conversely expresses low levels of ROS and high levels of NO, while both subsets express arginase [57]. The suppressive activity of the G-MDSCs was shown to be ARG1-dependent, in contrast to the STAT1- and iNOS-dependent mechanism of M-MDSCs [58].

Non-T-cell related mechanisms of suppression

While initially described as merely T-cell suppressive, emerging evidence suggests that MDSCs also interact with and modulate the function of other immune cells, particularly including macrophages [59], NK cells $[60,61]$, and Tregs [61]. Moreover, MDSCs, tumour-associated macrophages (TAMs) and DCs have been reported to interact and to cross-promote their immunosuppressive activities within the tumour microenvironment [62]. MDSCs in the tumour microenvironment were described to rapidly differentiate into TAMs through a HIF-1 $\alpha$ mediated mechanism [31]. MDSCs, in turn, producing high levels of IL-10, downregulate macrophage IL-12 production, promote TAMs and macrophage M2 polarisation and facilitate the development of Tregs [59, 63]. Furthermore, regulatory DCs (regDCs) have been described in cancer contexts as distinct DC subpopulation, which directly inhibit effector T-cells and indirectly induce or activate Treg cells and MDSCs [64]. Mechanistically, the immunosuppressive effects of regDCs were found to be mediated through IL-10, TGF- $\beta$, COX-2, iNOS, arginase and indoleamine 2,3-dioxygenase (IDO) [65-67].

MDSC plasticity/fibrocytic MDSCS

Recent studies suggest that $\mathrm{Grl}^{+}$myeloid-derived monocytic cells and MDSCs can transdifferentiate into extracellular matrix (collagen type I)-producing fibrocytes, a mechanism involving $\mathrm{CD} 4^{+} \mathrm{T}$-cells, IL-2, IL-4, IFN- $\gamma$ and TNF, GM-CSF/G-CSF, Kruppel-like factor 4 and fibroblast-specific protein 1 [68-70]. Fibrocytic MDSCs were found to interact with activated T-cells in a cell contact dependent manner, resulting in the production of IDO and leading to Treg expansion [69]. Fibrocytes can migrate into the tumour stroma microenvironment and further differentiate into myofibroblasts and promote tumorigenesis [71, 72] as well as metastasis [73]. Targeting fibrocytic MDSCs could represent a strategy to prevent the formation of the pre-metastatic niches and subsequently suppress metastasis formation.

\section{Other mechanisms}

Less established mechanisms used by MDSCs to suppress immune responses include: 1) upregulation of cyclooxygenase 2 and $\mathrm{PGE}_{2}[16]$; 2) secretion of TGF- $\beta$ [22]; and 3) sequestering cysteine as well as limiting the availability of cysteine, which is an essential amino acid for T-cell activation and proliferation [74]. Several studies demonstrate that the immunosuppressive functions of MDSCs require cell-cell contact, suggesting that MDSCs act through cell-surface receptors and/or the release of short-lived paracrine mediators [7]. MDSCs produce the anti-inflammatory cytokine IL-10 and dampen both CD4 ${ }^{+}$T-cells and NK cell responses [7, 75, 76], while promoting the expansion of Treg [63] and M2-like macrophages [59].

MDSCs in lung diseases

Some of the different lung diseases in which MDCs play a role and the mechanisms that are used are shown in figure 1. 


\section{Lung cancer and lung metastasis}

$\mathrm{T}$ and NK cells are essential for tumour elimination in the lung [77]. Accordingly, factors that regulate their activity are of high interest for lung cancer treatment strategies. Accumulating evidence suggest that MDSCs are responsible for inhibiting host T-cell activity against tumour-associated antigens and consequently impair the effectiveness of anti-cancer immunotherapeutic approaches [78]. MDSCs numbers were found to be associated inversely with responsiveness to chemotherapy and positively with shorter survival in patients with lung cancer $[79,80]$. Several studies support the concept that MDSCs dampen T-cells in lung cancer through direct contact and through mechanisms involving a plethora of mediators and mechanisms, such as iNOS, ARG1, TGF- $\beta$, IL-10 and the induction of Tregs [55, 75, 81-86]. MDSCs are recruited to the tumour site by the CC chemokine ligand (CCL) 2, CXC chemokine ligand (CXCL) 12, and CXCL5 [87]. The tumour microenvironment stimulates MDSCs to acquire immunosuppressive properties, which are mediated through STAT1, STAT3, STAT6 and nuclear factor $\mathrm{\kappa B}$ transcription factors [7]. Activated MDSCs, in turn, produce ARG1, iNOS2, IDO, NADPH oxidase and immunosuppressive cytokines that have the potential to inhibit cytotoxic T lymphocytes, DC, and NK cells as well as expand CD4 ${ }^{+} \mathrm{CD} 25^{+}$FoxP3 ${ }^{+}$Tregs $[88,89]$.

Many tumour types show an organ tropism of metastatic outgrowth, which was first proposed by Stephen Paget's seed and soil theory in 1889 [90]. Colon cancer, as an example, induces predominantly metastasis in the lung. A decade ago KAPLAN et al. [91] first described the concept of the premetastatic niches appearing in the lung as target organ. Signalling factors and cytokines of the primary tumour, e.g. vascular endothelial growth factor, placental growth factor, lysyl oxidases, and TNF or TGF- $\beta$, lead to the recruitment of immature bone marrow derived cells, which mainly consist of G-MDSCs, and form pre-metastatic niches in organs distinct from the location of the primary tumour. Within the pre-metastatic niche the main drivers for the infiltration of circulating CXC receptor (CXCR) $4^{+}$tumour cells are the remodelling of the extracellular matrix by matrix metalloproteinase 9 (MMP9), expression of the adhesion molecule fibronectin, pro-inflammatory S100A8/9 signalling and finally the release of the chemokine stromal cell-derived factor 1 , the agonist for CXCR4 [91]. Also hypoxia of the primary breast tumour accompanied by angiogenesis signalling promotes the infiltration of G-MDSCs with potent immunosuppression of NK cells [92].

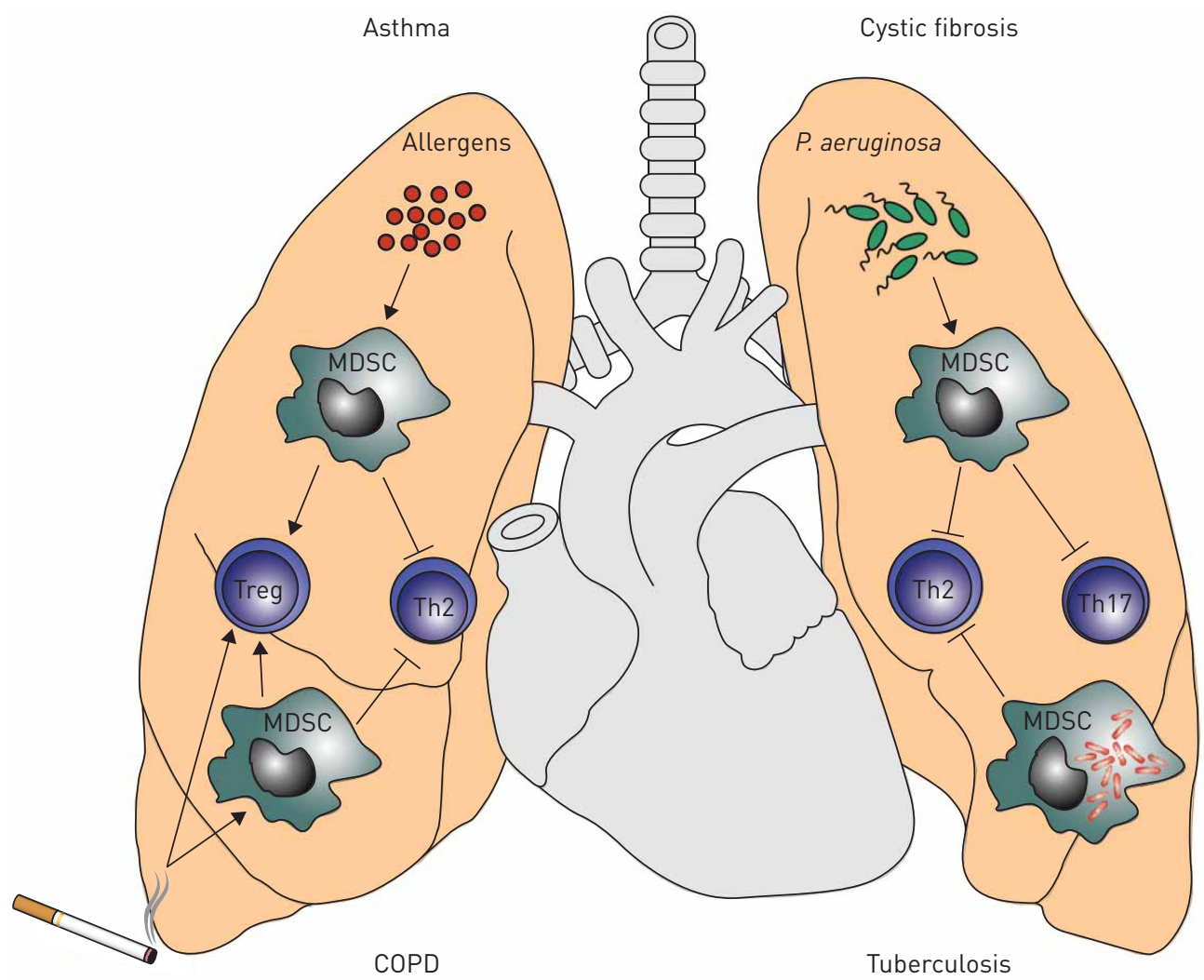

FIGURE 1 The role of myeloid-derived suppressor cells in lung diseases. In response to allergens (asthma), cigarette smoke (chronic obstructive pulmonary disease (COPD)), P. aeruginosa bacteria (cystic fibrosis) or Mycobacterium tuberculosis bacteria (tuberculosis), myeloid-derived suppressor cells (MDSCs) accumulate in the lungs and cooperate with regulatory T-cells (Tregs) to suppress T-helper type 2 (Th2) cells or Th17-driven inflammation. In tuberculosis, MDSCs phagocytose the mycobacteria and dampen surrounding T-cell responses, thereby supporting immune-evasion. 
Data from animal lung cancer models showed that COX-2 is involved in MDSC regulation through the production of PGE2 and ARG1 [43]. SRIVASTAVA et al. [93] demonstrated that Snail, an activating transcription factor in epithelial-mesenchymal transition, induces tumour growth and metastasis by increasing MDSCs via increasing intracellular expression of ARG1 in murine lung tumour microenvironment. In other murine models of lung cancer, targeting of MDSCs using antibodies improved antitumor activity via enhancing effector and memory T-cell responses, as well as NK cell and antigen-presenting cell activities [94-96]. Peripheral blood MDSCs levels were shown to correlate with a higher tumour burden and a worse prognosis [97-99]. Several approaches have been pursued to eliminate MDSCs in cancer, particularly abrogation of MDSCs using all-trans retinoic acid [100, 101], chemotherapy (gemcitabine, 5-fluoro-uracil) [102, 103], MMP9 inhibition (amino-biphosphonates) [104], MDSC proliferation inhibitors such as tyrosine kinase inhibitors (sunitinib and sorafinib) [97, 105, 106], MDSC recruitment inhibitors (CXCR2 antagonists) [109], MDSC function/activation inhibitors such as COX-2 inhibitors [16, 43, 108] and phosphodiesterase-5 inhibitors (sildanefil) [109]. Another novel approach to abrogate MDSCs, so called "peptibody" treatment showed complete depletion of blood, splenic, and intratumoural G- and M-MDSCs in tumour bearing mice, without affecting pro-inflammatory immune cell types [110]. Based on these studies, the translational and therapeutic potential of targeting MDSCs, in combination with conventional therapies, could be a promising approach for future anti-cancer therapy in human patients. Overall, the development of novel therapeutic agents that eliminate the activity of MDSCs in human lung cancer should accelerate our understanding of their biological role within the tumour microenvironment.

\section{Asthma}

$\mathrm{CD} 11 \mathrm{~b}^{+} \mathrm{Gr} 1^{\text {int }} \mathrm{F} 4 / 80^{+} \mathrm{MDSC}$-like cells were found to accumulate in allergic asthma and to suppress lung DC-mediated reactivation of primed Th2 cells in a toll-like receptor (TLR) 4- and MyD88-dependent fashion, mediated by IL-10 and ARG1 [75, 111]. It has been further shown that tumour-derived MDSCs suppress Th2-dominant inflammation in asthmatic mice, reduce recruitment of inflammatory cells and suppress production of IgE and Th2 cytokines in a TGF- $\beta 1$ dependent manner [112]. The chemokine CCL2 was found to recruit MDSCs into lung tissues in airway inflammation [112]. Aspirin treatment dampened the accumulation of G-MDSCs in the inflamed lung accompanied by increased Th2 airway responses [113]. It has further been demonstrated that $\mathrm{COX}$ and its product, $\mathrm{PGE}_{2}$, play an important role in the regulation of activation and accumulation of MDSCs through $\mathrm{PGE}_{2}$ and $\mathrm{PGE}_{4}$ receptors $[113,114]$. MDSCs and IL-10 levels significantly increased and negatively correlated with IL-12 levels during the onset of asthma in both human and mice. IL-10 not only suppressed the production of pro-inflammatory factors by macrophages, but also reduced cytotoxic effects and decreased NO production. Therefore, it is proposed that MDSCs play a dual role in asthma by upregulating anti-inflammatory IL-10 and downregulating pro-inflammatory IL-12 [76]. In a different study, MDSCs enhanced mast cell-mediated secretion of several inflammatory cytokines, including TNF, IL-6, IL-13, macrophage inflammatory protein-1 $\alpha$ and monocyte chemoattractant protein-1. The mutual interaction of MDSCs and mast cells enhanced the activities of each cell type, resulting in exacerbated inflammation and airway hyperresponsiveness [115]. Three different populations of $\mathrm{CD}_{11} \mathrm{~b}^{+} \mathrm{Ly}-6 \mathrm{G}^{+}$myeloid cells infiltrated the lung in a mouse model of allergic airway inflammation where they differentially generate the reactive free radicals $\mathrm{NO}$ and $\mathrm{O}_{2}^{-}$. The Ly-6C $\mathrm{Cy}^{+} 6 \mathrm{G}^{-}$ subset (predominant $\mathrm{NO}$ producer) and the $\mathrm{Ly}-6 \mathrm{C}^{+} \mathrm{Ly}-6 \mathrm{G}^{+}$subset were found to suppress $\mathrm{T}$-cell proliferation. In contrast, the $\mathrm{O}_{2}^{--}$that generates the Ly- $6 \mathrm{C}^{-} \mathrm{Ly}-6 \mathrm{G}^{+}$subset enhanced T-cell responses [116]. Superoxide-producing myeloid-derived regulatory cells (MDRCs), present in high numbers in the airways of patients with mild asthma or chronic obstructive pulmonary disease (COPD), but not in healthy controls, were, on the other hand, found to enhance proliferation of CD4 ${ }^{+}$T-cells [117].

\section{COPD}

NO producing MDRSCs were found in the airways of patients with mild asthma, but not in COPD patients or healthy control individuals and, were found to suppress activated CD4 ${ }^{+}$T-cells [117]. Smoking upregulated and activated circulating MDSCs in COPD patients, but not in smokers with normal lung function [118]. In COPD patients the MDSC activation was accompanied by down-regulation of the T-cell receptor $\zeta$ chain expression in T-cells [118]. In addition, it has been shown that MDSCs were elevated in the bone marrow, spleens, and lungs after 4 months of cigarette smoke exposure, while this was paralleled by decreased pulmonary DCs [119]. However, these phenotypic MDSCs lacked immune suppressive activity, and thus were not bona fide MDSCs [119]. In a further study, MDSCs were also increased in patients with COPD and correlated with elevated levels of Tregs, which is in agreement with studies that suggest reciprocal control of these two cell types [120]. In summary, these studies suggest that the accumulation of MDSCs in COPD may underlie the blunted immune response observed in COPD. 


\section{Tuberculosis}

Development of active tuberculosis (TB) is known to correlate with impaired T-cell responses, but the underlying immune mechanisms remained incompletely understood [121-125]. Both patients with acute (household exposure within 3 months) and chronic TB were recently described to show significantly higher frequencies of MDSCs that inhibited functions of $\mathrm{CD}^{+}$and $\mathrm{CD} 8^{+} \mathrm{T}$-cells, including $\mathrm{T}$-cell proliferation, altered T-cell trafficking as well as production of IL-2, IFN- $\gamma$ and TNF [126]. The frequency of $\mathrm{CD}^{-} \mathrm{CD} 244^{\text {high }}$ cells with MDSC phenotypes were significantly higher in active TB patients and were inversely associated with the activation and functionality of $\mathrm{CD}^{+}$and $\mathrm{CD} 8^{+} \mathrm{T}$-cells [127]. In murine experimental pulmonary TB, MDSCs readily phagocytosed Mycobacterium tuberculosis, and released both pro-inflammatory (IL-6, IL-1 $\alpha$ ) and immunomodulatory (IL-10) cytokines, while retaining their suppressive capacity [128]. Excessive MDSC accumulation in lungs correlated with elevated surface expression of IL-4R $\alpha$ and increased TB lethality, whereas targeted depletion of MDSCs ameliorated disease in this animal TB model [128]. In summary, these findings indicate that MDSCs accumulate in lungs during pulmonary TB and play a dual role in host-pathogen interaction: MDSCs phagocytose and harbour M. tuberculosis bacteria intracellularly, providing a cellular shelter, while simultaneously dampening surrounding hostile T-cell responses.

\section{Pulmonary hypertension}

Pulmonary hypertension is a progressive syndrome with dysregulated inflammatory processes [129]. Immunohistochemical analysis of lung sections from patients with pulmonary hypertension indicated that immature DCs are present in peribronchovascular regions of vascular remodelling [130]. In a rat model of monocrotaline-induced pulmonary hypertension, DCs with immature myeloid phenotype were recruited to remodelled vessels [130]. In addition, monocyte-derived DCs from patients with pulmonary hypertension were defective in their ability to stimulate T-cells in an allostimulatory mixed-leukocyte reaction assay. In this respect, abnormalities of $\mathrm{T}$ lymphocyte subsets have been documented in patients with pulmonary hypertension $[131,132]$. In pulmonary hypertension patients, circulating activated MDSC numbers were significantly increased in comparison to control subjects and correlated with increasing mean pulmonary artery pressure [133]. A direct mechanistic role for MDSCs in pulmonary hypertension and inflammation-associated vascular remodelling has not yet been defined.

\section{Cystic fibrosis}

Cystic fibrosis (CF) patients are impaired in eradicating $P$. aeruginosa infections and show skewed T-cell proliferation and immune responses, but the underlying reasons remained poorly understood [134-136]. Recently, it has been shown that G-MDSCs accumulate in CF patients, particularly in patients chronically infected with $P$. aeruginosa and correlate with CF lung disease activity [28]. Flagellated $P$. aeruginosa induced MDSC generation, corresponding to TLR5 surface expression on G-MDSCs. Moreover, G-MDSCs in $\mathrm{CF}$ patients were further characterised by an upregulation of the chemokine receptor and HIV-coreceptor CXCR4 on the surface of MDSCs. Functionally, both CF patient-isolated and flagellin-induced MDSCs suppressed T-cell proliferation and modulated Th17 cells, as key antibacterial T-cell populations in CF. Percentages of circulating G-MDSCs correlated with pulmonary function in CF patients chronically infected with $P$. aeruginosa. MDSCs could, therefore, represent a novel therapeutic target in CF patients, particularly in patients chronically infected with P. aeruginosa [26].

\section{Pulmonary infection}

Efficient innate host defence is crucial for the elimination of invading pulmonary pathogens [137, 138], but uncontrolled immune activation leads to collateral tissue damage. Neutrophilic cells that are rapidly recruited to the site of infection produce ROS and proteases to clear infection. However, due to a relatively short life span, neutrophils rapidly undergo apoptosis, secondary necrosis or neutrophil extracellular trap formation at the pulmonary site of infection. MDSCs have been described to efficiently efferocytose apoptotic neutrophils, mediated by IL-10 [139]. Clearance of dead neutrophils by MDSCs may, therefore help to resolve lung inflammation, preventing lung injury and ultimately restore tissue homeostasis. Studies in Stat $^{-/-}$mice showed that bacterial infection significantly increased pulmonary MDSCs, while decreasing neutrophils [139]. These observations suggest that increasing MDSCs via STAT1 inhibition in combination with effective antibiotic therapy may be beneficial in the context of non-resolving bacterial pneumonia. On the other hand, it has been shown that expansion of MDSCs and absence of invariant NK T-cells in influenza A infection suppresses influenza-specific immune responses [140]. These paradoxical effects in viral and bacterial infection may be due to different MDSC kinetics and/or downstream responses [139, 140]. MDSCs accumulate in the lungs during pneumocystis pneumonia (PCP) [141]. At the pulmonary site, MDSCs interact with alveolar macrophages through programmed cell death protein 1 and programmed death-ligand 1, leading to macrophage suppression through histone modification and DNA methylation of the PU.1 gene, finally resulting in PU.1 downregulation. MDSCs employ the same 
mechanism to interact with monocytes, leading to PU.1 downregulation and inhibition of their differentiation into alveolar macrophages, resulting in decreased numbers and activity of alveolar macrophages during PCP [142].

\section{Pulmonary inflammation}

Exposure of mice to lipopolysaccharide (LPS) triggers the recruitment of a MDSC-like phenotype

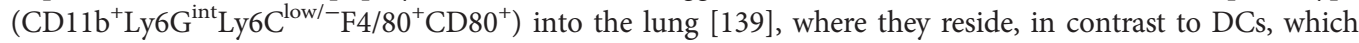
traffic readily to the lymph nodes [75]. LPS-induced lung MDSCs were further shown to blunt the ability of pulmonary DCs to promote Th2 responses [75, 143-145]. It is speculated that lung MDSCs compromise Th2 cell survival, thereby reducing the size of the memory T-cell pool [75, 146, 147]. Thus, collectively, it appears that an important effector function of TLR-induced MDSCs is not directed to the lymph node to influence the development of adaptive immune functions, but rather to control local pulmonary immune responses. Recent studies further suggest that TLR4 activation by LPS induces GM-CSF and IL-6 production leading to STAT5 and STAT3 activation, which in turn drives MDSC generation [82, 139, 148, 149]. At the cellular level, MDSCs induce Tregs [150] by increasing Foxp3 expression through an IL-10-, TGF- $\beta$ - and ARG1-dependent mechanism [150]. It has been further shown that glucocorticoids induce a distinct anti-inflammatory phenotype in mouse monocytes, which phenotypically resemble MDSCs with respect to the expression of CD11b, Ly-6G and IL-4R $\alpha$ chain [151].

\section{Conclusions and outlook}

Emerging evidence suggests that MDSCs, as immuosuppressive myeloid cells, play a critical role in malignant, infectious and inflammatory lung diseases, particularly lung cancer, TB, COPD, pulmonary hypertension, asthma and CF. Amongst the MDSC subsets, granulocytic MDSCs appear to represent the major population accumulating in pulmonary diseases. Pathways orchestrating MDSC generation, recruitment, activation and suppressive functions are diverse and future studies are required to narrow down the most relevant ones for therapeutic targeting approaches. Mechanistically, a more precise understanding of how host- or pathogen-derived cues modulate MDSC generation and function will help to develop tailored MDSC inhibitors for conditions where MDSC cause harm to the host, such as lung cancer and pulmonary infection (for instance TB), where pulmonary MDSCs favour the survival of malignant cells or pathogens. Conversely, adoptive cellular transfer or specific activation of MDSCs may represent an attractive therapeutic strategy to dampen immune responses in the setting of immune over activation, as found in allergic, autoimmune and auto-inflammatory pulmonary disease conditions. Findings from both mouse models and human patients indicate a potential therapeutic role for vitamin $\mathrm{A}$ and $\mathrm{D}$, tyrosine kinase inhibitors, chemokine receptor antagonists, COX inhibitors and phosphodiesterase-5 inhibitors in regulating MDSCs. Clinical interventional studies are the next consequent step to systematically assess the safety and efficacy of these MDSC-interfering approaches in pulmonary diseases.

\section{Acknowledgements}

We thank Peter M. Weber, University of Tübingen (Tübingen, Germany) for the excellent illustration.

\section{References}

Strober S, Okada S, Oseroff A. Role of natural suppressor cells in allograft tolerance. Fed Proc 1984; 43: 263-265. Buessow SC, Paul RD, Lopez DM. Influence of mammary tumor progression on phenotype and function of spleen and in situ lymphocytes in mice. J Natl Cancer Inst 1984; 73: 249-255.

3 Young MR, Endicott RA, Duffie GP, et al. Suppressor alveolar macrophages in mice bearing metastatic Lewis lung carcinoma tumors. J Leukoc Biol 1987; 42: 682-688.

4 Talmadge JE, Gabrilovich DI. History of myeloid-derived suppressor cells. Nat Rev Cancer 2013; 13: 739-752.

5 Saleem SJ, Conrad DH. Hematopoietic cytokine-induced transcriptional regulation and notch signaling as modulators of MDSC expansion. Int Immunopharmacol 2011; 11: 808-815.

6 Gabrilovich DI. Editorial: The intricacy of choice: can bacteria decide what type of myeloid cells to stimulate? J Leukoc Biol 2014; 96: 671-674.

7 Gabrilovich DI, Nagaraj S. Myeloid-derived suppressor cells as regulators of the immune system. Natur Rev Immunol 2009; 9: 162-174.

8 Cripps JG, Gorham JD. MDSC in autoimmunity. Int Immunopharmacol 2011; 11: 789-793.

9 Dumitru CA, Moses K, Trellakis S, et al. Neutrophils and granulocytic myeloid-derived suppressor cells: immunophenotyping, cell biology and clinical relevance in human oncology. Cancer Immunol Immunother 2012; 61: 1155-1167.

10 Youn JI, Nagaraj S, Collazo M, et al. Subsets of myeloid-derived suppressor cells in tumor bearing mice. J Immunol 2008; 181: 5791-5802.

11 Youn JI, Collazo M, Shalova IN, et al. Characterization of the nature of granulocytic myeloid-derived suppressor cells in tumor-bearing mice. J Leukoc Biol 2012; 91: 167-181.

12 Gallina G, Dolcetti L, SeraWni P, et al. Tumors induce a subset of inflammatory monocytes with immunosuppressive activity on CD8 + T cells. J Clin Invest 2006; 116: 2777-2790.

13 Rößner S, Voigtländer C, Wiethe C, et al. Myeloid dendritic cell precursors generated from bone marrow suppress $\mathrm{T}$ cell responses via cell contact and nitric oxide production in vitro. Eur J Immunol 2005; 35: 3533-3544. 
Mazzoni A, Bronte V, Visintin A, et al. Myeloid suppressor lines inhibit T cell responses by an NO-dependent mechanism. J Immunol 2002; 168: 689-695.

Pan PY, Wang GX, Yin B, et al. Reversion of immune tolerance in advanced malignancy: modulation of myeloid derived suppressor cell development by blockade of stem-cell factor function. Blood 2008; 111: 219-228.

Sinha P, Clements VK, Fulton AM, et al. Prostaglandin E2 promotes tumor progression by inducing myeloid-derived suppressor cells. Cancer Res 2007; 67: 4507-4513.

Serafini P, Carbley R, Noonan KA, et al. High-Dose GM-CSF-producing vaccines impair the immune response through the recruitment of myeloid suppressor cells. Cancer Res 2004; 64: 6337-6343.

Young MR, Wright MA, Coogan M, et al. Tumor-derived cytokines induce bone marrow suppressor cells that mediate immunosuppression through transforming growth factor beta. Cancer Immunol Immunother 1992; 35: 14-18.

Maeda H, Shiraishi A. TGF-beta contributes to the shift toward Th2-type responses through direct and IL-10-mediated pathways in tumor-bearing mice. J Immunol 1996; 156: 73-78.

Alleva DG, Walker TM, Elgert KD. Induction of macrophage suppressor activity by fibrosarcoma-derived transforming growth factor-beta 1: contrasting effects on resting and activated macrophages. J Leukoc Biol 1995; 57: 919-928.

Yang L, Huang J, Ren X, et al. Abrogation of TGF beta signaling in mammary carcinomas recruits Gr-1+CD11b +myeloid cells that promote metastasis. Cancer Cell 2008; 13: 23-35.

Tu S, Bhagat G, Cui G, et al. Overexpression of interleukin-1beta induces gastric inflammation and cancer and mobilizes myeloid-derived suppressor cells in mice. Cancer Cell 2008; 14: 408-419.

Foell D, Wittkowski H, Vogl T, et al. S100 proteins expressed in phagocytes: a novel group of damage-associated molecular pattern molecules. J Leukoc Biol 2007; 81: 28-37.

Sinha P, Okoro C, Foell D, et al. Proinflammatory S100 proteins regulate the accumulation of myeloid-derived suppressor cells. J Immunol 2008; 181: 4666-4675.

Lechner MG, Liebertz DJ, Epstein AL. Characterization of cytokine-induced myeloid-derived suppressor cells from normal human peripheral blood mononuclear cells. J Immunol 2010; 185: 2273-2284.

Li L, Zhang J, Diao W, et al. MicroRNA-155 and MicroRNA-21 promote the expansion of functional myeloid-derived suppressor cells. J Immunol 2014; 192: 1034-1043.

Rieber N, Brand A, Hector A, et al. Flagellin induces myeloid-derived suppressor cells: implications for Pseudomonas aeruginosa infection in cystic fibrosis lung disease. J Immunol 2013; 190: 1276-1284.

Rieber N, Singh A, Öz H, et al. Pathogenic fungi regulate immunity by inducing neutrophilic myeloid-derived suppressor cells. Cell Host Microbe 2015; 17: 507-514.

Youn JI, Kumar V, Collazo M, et al. Epigenetic silencing of retinoblastoma gene regulates pathologic differentiation of myeloid cells in cancer. Nat Immunol 2013; 14: 211-220.

Corzo CA, Condamine T, Lu L, et al. HIF- $1 \alpha$ regulates function and differentiation of myeloid-derived suppressor cells in the tumor microenvironment. J Exp Med 2010; 207: 2439-2453.

Condamine T, Kumar V, Ramachandran IR, et al. ER stress regulates myeloid-derived suppressor cell fate through TRAIL-R-mediated apoptosis. J Clin Invest 2014; 124: 2626-2639. IFN- $\gamma$ treatment impairs DC development. Eur J Immunol 2009; 39: 2865-2876.

Rieber N, Gille C, Köstlin N, et al. Neutrophilic myeloid derived suppressor cells in cord blood modulate innate and adaptive immune responses. Clin Exp Immunol 2013; 174: 45-52.

Dietlin TA, Hofman FM, Lund BT, et al. Mycobacteria-induced Gr-1+ subsets from distinct myeloid lineages have opposite effects on T cell expansion. J Leukoc Biol 2007; 81: 1205-1212.

Szuster-Ciesielska A, Hryciuk-Umer E, Stepulak A, et al. Reactive oxygen species production by blood neutrophils of patients with laryngeal carcinoma and antioxidative enzyme activity in their blood. Acta Oncol 2004; 43: 252-258.

Waris G, Ahsan H. Reactive oxygen species: role in the development of cancer and various chronic conditions. J Carcinog 2006; 5: 14.

Mantovani G, Macciò A, Madeddu C, et al. Antioxidant agents are effective in inducing lymphocyte progression through cell cycle in advanced cancer patients: assessment of the most important laboratory indexes of cachexia and oxidative stress. J Mol Med 2003; 81: 664-673.

Agostinelli E, Seiler N. Non-irradiation-derived reactive oxygen species (ROS) and cancer: therapeutic implications. Amino Acids 2006; 31: 341-355.

Kusmartsev S, Nefedova Y, Yoder D, et al. Antigen-specific inhibition of CD8+ T cell response by immature myeloid cells in cancer is mediated by reactive oxygen species. J Immunol 2004; 172: 989-999.

Sauer H, Wartenberg M, Hescheler J. Reactive oxygen species as intracellular messengers during cell growth and differentiation. Cell Physiol Biochem 2001; 11: 173-186. 641-654.

Rodríguez PC, Ochoa AC. Arginine regulation by myeloid derived suppressor cells and tolerance in cancer: mechanisms and therapeutic perspectives. Immunol Rev 2008; 222: 180-191.

Rodriguez PC, Hernandez CP, Quiceno D, et al. Arginase I in myeloid suppressor cells is induced by COX-2 in lung carcinoma. J Exp Med 2005; 202: 931-939.

Rodriguez PC, Zea AH, Culotta KS, et al. Regulation of T cell receptor CD3zeta chain expression by L-arginine. J Biol Chem 2002; 277: 21123-21129.

Rodriguez PC, Quiceno DG, Ochoa AC. L-arginine availability regulates T-lymphocyte cell-cycle progression. Blood 2007; 109: 1568-1573.

Bingisser R, Tilbrook $\mathrm{P}$, Holt $\mathrm{P}$, et al. Macrophage-derived nitric oxide regulates $\mathrm{T}$ cell activation via reversible disruption of the Jak3/STAT5 signaling pathway. J Immunol 1998; 160: 5729-5734.

Harari O, Liao JK. Inhibition of MHC II gene transcription by nitric oxide and antioxidants. Curr Pharm 2004; 10: 893-898.

Rivoltini L, Carrabba M, Huber V, et al. Immunity to cancer: attack and escape in T lymphocyte-tumor cell interaction. Immunol Rev 2002; 188: 97-113. 

oxide synthase and nitrotyrosine with fibroblast growth factor transformation in pancreatic cancer. Arch Surg 1999; 134: 245-251.

50 Cobbs CS, Whisenhunt TR, Wesemann DR, et al. Inactivation of wild-type p53 protein function by reactive oxygen and nitrogen species in malignant glioma cells. Cancer Res 2003; 63: 8670-8673.

51 Bentz BG, Haines GK III, Radosevich JA. Increased protein nitrosylation in head and neck squamous cell carcinogenesis. Head Neck 2000; 22: 64-70.

52 Dairou J, Dupret JM, Rodrigues-Lima F. Impairment of the activity of the xenobiotic-metabolizing enzymes arylamine $\mathrm{N}$-acetyltransferases 1 and 2 (NAT1/NAT2) by peroxynitrite in mouse skeletal muscle cells. FEBS Lett 2005; 579: 4719-4723.

53 Ekmekcioglu S, Ellerhorst J, Smid CM, et al. Inducible nitric oxide synthase and nitrotyrosine in human metastatic melanoma tumors correlate with poor survival. Clin Cancer Res 2000; 6: 4768-4775.

54 Kinnula VL, Torkkeli T, Kristo P, et al. Ultrastructural and chromosomal studies on manganese superoxide dismutase in malignant mesothelioma. Am J Respir Cell Mol Biol 2004; 31: 147-153.

55 Yang R, Cai Z, Zhang Y, et al. CD80 in immune suppression by mouse ovarian carcinoma-associated Gr-1 +CD11b+ myeloid cells. Cancer Res 2006; 66: 6807-6815.

56 Huang B, Pan PY, Li Q, et al. Gr-1+CD115+ immature myeloid suppressor cells mediate the development of tumor induced T regulatory cells and T-cell anergy in tumor-bearing host. Cancer Res 2006; 66: 1123-1131.

57 Zhu B, Bando Y, Xiao S, et al. CD11b+Ly-6Chi Suppressive Monocytes in Experimental Autoimmune Encephalomyelitis. J Immunol 2007; 179: 5228-5237.

58 Movahedi K, Guilliams M, Van den Bossche J, et al. Identification of discrete tumor-induced myeloid-derived suppressor cell subpopulations with distinct T-cell suppressive activity. Blood 2008; 111: 4233-4244.

59 Sinha P, Clements VK, Bunt SK, et al. Crosstalk between myeloid derived suppressor cells and macrophages subverts tumor immunity toward a type 2 response. J Immunol 2007; 179: 977-983.

60 Shime H, Kojima A, Maruyama A, et al. Myeloid-derived suppressor cells confer tumor-suppressive functions on natural killer cells via polyinosinic:polycytidylic acid treatment in mouse tumor models. J Innate Immun 2014; 6: 293-305.

61 Lindau D, Gielen P, Kroesen M, et al. The immunosuppressive tumour network: myeloid-derived suppressor cells, regulatory T cells and natural killer T cells. Immunology 2013; 138: 105-115.

62 Ostrand-Rosenberg S, Sinha P, Beury DW, et al. Cross-talk between myeloid derived suppressor cells (MDSC), macrophages, and dendritic cells enhances tumor induced immune suppression. Semin Cancer Biol 2012; 22: 275-281.

63 Murai M, Turovskaya $\mathrm{O}$, Kim G, et al. Interleukin 10 acts on regulatory $\mathrm{T}$ cells to maintain expression of the transcription factor Foxp3 and suppressive function in mice with colitis. Nat Immunol 2009; 10: 1178-1184.

64 Ma Y, Shurin GV, Gutkin DW, et al. Tumor associated regulatory dendritic cells. Semin Cancer Biol 2012; 22: 298-306.

65 Shurin MR, Naiditch H, Zhong H, et al. Regulatory dendritic cells: new targets for cancer immunotherapy. Cancer Biol Ther 2011; 11: 988-992.

66 Shurin GV, Ouellette CE, Shurin MR. Regulatory dendritic cells in the tumor immunoenvironment. Cancer Immunol Immunother 2012; 61: 223-230.

67 Liu Q, Zhang C, Sun A, et al. Tumor-educated CD11b ${ }^{\text {high }} \mathrm{la}^{\text {low }}$ regulatory dendritic cells suppress $\mathrm{T}$ cell response through arginase I. J Immunol 2009; 182: 6207-6216.

68 Niedermeier M, Reich B, Rodriguez Gomez M, et al. CD4+ T cells control the differentiation of Gr1+ monocytes into fibrocytes. Proc Natl Acad Sci USA 2009; 106: 17892-17897.

69 Zoso A, Mazza EM, Bicciato S, et al. Human fibrocytic myeloid-derived suppressor cells express IDO and promote tolerance via Treg-cell expansion. Eur J Immunol 2014; 44: 3307-3319.

70 Shi Y, Ou L, Han S, et al. Deficiency of Kruppel-like factor KLF4 in myeloid-derived suppressor cells inhibits tumor pulmonary metastasis in mice accompanied by decreased fibrocytes. Oncogenesis 2014; 3: e129.

71 Hong KM, Belperio JA, Keane MP, et al. Differentiation of human circulating fibrocytes as mediated by transforming growth factor-beta and peroxisome proliferator-activated receptor gamma. J Biol Chem 2007; 282: 22910-22920.

72 Mehner C, Radisky DC. Triggering the landslide: the tumor-promotional effects of myofibroblasts. Exp Cell Res 2013; 319: 1657-1662.

73 van Deventer HW, Palmieri DA, Wu QP, et al. Circulating fibrocytes prepare the lung for cancer metastasis by recruiting Ly-6C+ monocytes via CCL2. J Immunol 2013; 190: 4861-4867.

74 Srivastava MK, Sinha P, Clements VK, et al. Myeloid derived suppressor cells inhibit T-cell activation by depleting cystine and cysteine. Cancer Res 2010; 70: 68-77.

75 Arora M, Poe SL, Oriss TB, et al. TLR4/MyD88-induced CD11b+Gr-1intF4/80+ non-migratory myeloid cells suppress Th2 effect or function in the lung. Mucosal Immunol 2010; 3: 578-593.

76 Zhang YL, Luan B, Wang XF, et al. Peripheral blood MDSCs, IL-10 and IL-12 in children with asthma and their importance in asthma development. PLoS One 2013; 8: e63775.

77 Borghaei H, Smith MR, Campbell KS. Immunotherapy of cancer. Eur J Pharmacol 2009; 625: 41-54

78 Nagaraj S, Gupta K, Pisarev V, et al. Altered recognition of antigen is a mechanism of CD8+ T cell tolerance in cancer. Nat Med 2007; 13: 828-835.

79 Feng PH, Lee KY, Chang YL, et al. CD14(+)S100A9(+) monocytic myeloid derived suppressor cells and their clinical relevance in non-small cell lung cancer. Am J Respir Crit Care Med 2012; 186: 1025-1036.

80 Liu CY, Wang YM, Wang CL, et al. Population alterations of L-arginase and inducible nitric oxide synthase-expressed CD11b+/CD14-/CD15+/ CD33+ myeloid-derived suppressor cells and CD8+ T lymphocytes in patients with advanced-stage non-small cell lung cancer. J Cancer Res Clin Oncol 2010; 136: 35-45.

81 Bronte V, Serafini P, De Santo C, et al. IL-4-induced arginase 1 suppresses alloreactive T cells in tumor-bearing mice. J Immunol 2003; 170: 270-278.

82 Condamine T, Gabrilovich DI. Molecular mechanisms regulating myeloid-derived suppressor cell differentiation and function. Trends Immunol 2011; 32: 19-25.

83 Bogdan C. Regulation of lymphocytes by nitric oxide. Methods Mol Biol 2011; 677: 375-393. 
Zea AH, Rodriguez PC, Atkins MB, et al. Arginase-producing myeloid suppressor cells in renal cell carcinoma patients: a mechanism of tumor evasion. Cancer Res 2005; 65: 3044-3048.

Liu Y, Van Ginderachter JA, Brys L, et al. Nitric oxide independent CTL suppression during tumor progression: association with arginase producing (M2) myeloid cells. J Immunol 2003; 170: 5064-5074.

Jackaman C, Nelson DJ. Are macrophages, myeloid derived suppressor cells and neutrophils mediators of local suppression in healthy and cancerous tissues in aging hosts? Exp Gerontol 2014; 54: 53-57.

Sawanobori Y, Ueha S, Kurachi M, et al. Chemokine-mediated rapid turnover of myeloid-derived suppressor cells in tumor-bearing mice. Blood 2008; 111: 5457-5466.

Gabrilovich DI, Ostrand-Rosenberg S, Bronte V. Coordinated regulation of myeloid cells by tumours. Nat Rev Immunol 2012; 12: 253-268.

Almand B, Clark JI, Nikitina E, et al. Increased production of immature myeloid cells in cancer patients: a mechanism of immunosuppression in cancer. J Immunol 2001; 166: 678-689.

Paget S. The distribution of secondary growths in cancer of the breast. Cancer Metastasis Rev 1989; 8: 98-101.

Kaplan RN, Riba RD, Zacharoulis S, et al. VEGFR1-positive haematopoietic bone marrow progenitors initiate the pre-metastatic niche. Nature 2005; 438: 820-827.

Sceneay J, Chow MT, Chen A, et al. Primary tumor hypoxia recruits CD11b+/Ly6Cmed/Ly6G+ immune suppressor cells and compromises NK cell cytotoxicity in the premetastatic niche. Cancer Res 2012; 72: 3906-3911.

Srivastava MK, Sharma S, Zhu L, et al. Abstract 17. Tumor Snail knockdown reduces tumor burden and metastases by inducing antitumor immune responses in lung cancer. Cancer Res 2011; 71: Suppl. 18, A17.

Sawant A, Schafer CC, Jin TH, et al. Enhancement of antitumor immunity in lung cancer by targeting myeloid-derived suppressor cell pathways. Cancer Res 2013; 73: 6609-6620.

Srivastava MK, Zhu L, Harris-White M, et al. Myeloid suppressor cell depletion augments antitumor activity in lung cancer. PLoS One 2012; 7: e40677.

Mundy-Bosse BL, Lesinski GB, Jaime-Ramirez AC, et al. Myeloid-derived suppressor cell inhibition of the IFN response in tumor-bearing mice. Cancer Res 2011; 71: 5101-5110.

Ko JS, Zea AH, Rini BI, et al. Sunitinib mediates reversal of myeloid-derived suppressor cell accumulation in renal cell carcinoma patients. Clin Cancer Res 2009; 15: 2148-2157.

Gabitass RF, Annels NE, Stocken DD, et al. Elevated myeloid-derived suppressor cells in pancreatic, esophageal and gastric cancer are an independent prognostic factor and are associated with significant elevation of the Th2 cytokine interleukin-13. Cancer Immunol Immunother 2011; 60: 1419-1430.

Montero AJ, Diaz-Montero CM, Kyriakopoulos CE, et al. Myeloid-derived suppressor cells in cancer patients: a clinical perspective. J Immunother 2012; 35: 107-115

Mirza N, Fishman M, Fricke I, et al. All-trans-retinoic acid improves differentiation of myeloid cells and immune response in cancer patients. Cancer Res 2006; 66: 9299-9307.

Kusmartsev S, Cheng F, Yu B, et al. All-trans-retinoic acid eliminates immature myeloid cells from tumor-bearing mice and improves the effect of vaccination. Cancer Res 2003; 63: 4441-4449.

Suzuki E, Kapoor V, Jassar AS, et al. Gemcitabine selectively eliminates splenic Gr-1+/CD11b+ myeloid suppressor cells in tumor-bearing animals and enhances antitumor immune activity. Clin Cancer Res 2005; 11: 6713-6721.

Ko HJ, Kim YJ, Kim YS, et al. A combination of chemoimmunotherapies can efficiently break selftolerance and induce antitumor immunity in a tolerogenic murine tumor model. Cancer Res 2007; 67: 7477-7486.

Melani C, Sangaletti S, Barazzetta FM, et al. Amino-biphosphonate-mediated MMP-9 inhibition breaks the tumor-bone marrow axis responsible for myeloid-derived suppressor cell expansion and macrophage infiltration in tumor stroma. Cancer Res 2007; 67: 11438-11446.

Ozao-Choy J, Ma G, Kao J, et al. The novel role of tyrosine kinase inhibitor in the reversal of immune suppression and modulation of tumor microenvironment for immune-based cancer therapies. Cancer Res 2009; 69: $2514-2522$.

Xin H, Zhang C, Herrmann A, et al. Sunitinib inhibition of Stat3 induces renal cell carcinoma tumor cell apoptosis and reduces immunosuppressive cells. Cancer Res 2009; 69: 2506-2513.

Yanagawa J, Walser TC, Zhu LX, et al. Snail promotes CXCR2 ligand-dependent tumor progression in non-small cell lung carcinoma. Clin Cancer Res 2009; 15: 6820-6829.

Talmadge JE, Hood KC, Zobel LC, et al. Chemoprevention bycyclooxygenase-2 inhibition reduces immature myeloid suppressor cell expansion. Int Immunopharmacol 2007; 7: 140-151.

Serafini P, Meckel K, Kelso M, et al. Phosphodiesterase-5 inhibition augments endogenous antitumor immunity by reducing myeloid-derived suppressor cell function. J Exp Med 2006; 203: 2691-2702.

Qin H, Lerman B, Sakamaki I, et al. Generation of a new therapeutic peptide that depletes myeloid-derived suppressor cells in tumor-bearing mice. Nat Med 2014; 20: 676-681.

Arora M, Poe SL, Ray A, et al. LPS-induced CD11b+Gr1(int)F4/80+ regulatory myeloid cells suppress allergen-induced airway inflammation. Int Immunopharmacol 2011; 11: 827-832.

2 Song C, Yuan Y, Wang XM, et al. Passive transfer of tumour-derived MDSCs inhibits asthma-related airway inflammation. Scand J Immunol 2014; 79: 98-104.

Shi M, Shi G, Tang J, et al. Myeloid-derived suppressor cell function is diminished in aspirin-triggered allergic airway hyperresponsiveness in mice. J Allergy Clin Immunol 2014; 134: 1163-1174.

Obermajer N, Muthuswamy R, Lesnock J, et al. Positive feedback between $\mathrm{PGE}_{2}$ and COX2 redirects the differentiation of human dendritic cells toward stable myeloid-derived suppressor cells. Blood 2011; 118: 5498-5505.

Morales JK, Saleem SJ, Martin RK, et al. Myeloid-derived suppressor cells enhance IgE-mediated mast cell responses. J Leukoc Biol 2014; 95: 643-650.

6 Deshane J, Zmijewski JW, Luther R, et al. Free radical-producing myeloid-derived regulatory cells: potent activators and suppressors of lung inflammation and airway hyperresponsiveness. Mucosal Immunol 2011; 4: 503-518.

7 Deshane JS, Redden DT, Zeng M, et al. Subsets of airway myeloid-derived regulatory cells distinguish mild asthma from chronic obstructive pulmonary disease. J Allergy ClinImmunol 2015; 135: 413-424.

Scrimini S, Pons J, Agustí A, et al. Differential effects of smoking and COPD upon circulating myeloid derived suppressor cells. Respir Med 2013; 107: 1895-1903.

rtiz ML, Lu L, Ramachandran I, et al. Myeloid-derived suppressor cells in the development of lung cancer. Cancer Immunol Res 2014; 2: 50-58. 
120 Kalathil SG, Lugade AA, Pradhan V, et al. T-regulatory cells and programmed death 1+ T cells contribute to effector T-cell dysfunction in patients with chronic obstructive pulmonary disease. Am J Respir Crit Care Med 2014; 190: 40-50

121 Jurado JO, Alvarez IB, Pasquinelli V, et al. Programmed death (PD)-1: PD-ligand 1/PD-ligand 2 pathway inhibits T cell effector functions during human tuberculosis. J Immunol 2008; 181: 116-125.

122 Lazar-Molnar E, Chen B, Sweeney KA Jr, et al.. Programmed death-1 (PD-1)-deficient mice are extraordinarily sensitive to tuberculosis. Proc Natl Acad Sci USA 2010; 107: 13402-13407.

123 Wang X, Cao Z, Jiang J, et al. Elevated expression of Tim-3 on CD8 T cells correlates with disease severity of pulmonary tuberculosis. J Infect 2011; 62: 292-300.

124 Qiu Y, Chen J, Liao H, et al. Tim-3-expressing CD4+ and CD8+ T cells in human tuberculosis (TB) exhibit polarized effector memory phenotypes and stronger anti-TB effector functions. PLoS Pathog 2012; 8: e1002984.

125 Jiang J, Wang X, Wang X, et al. Reduced CD27 expression on antigen-specific CD4+ T cells correlates with persistent active tuberculosis. I Clin Immunol 2010; 30: 566-573. du Plessis N, Loebenberg L, Kriel M, et al. Increased frequency of myeloid-derived suppressor cells during active tuberculosis and after recent Mycobacterium tuberculosis infection suppresses T-cell function. Am J Respir Crit Care Med 2013; 188: 724-732.

127 Yang B, Wang X, Jiang J, et al. Identification of CD244-expressing myeloid-derived suppressor cells in patients with active tuberculosis. Immunol Lett 2014; 158: 66-72.

128 Knaul JK, Jörg S, Oberbeck-Mueller D, et al. Lung-residing myeloid-derived suppressors display dual functionality in murine pulmonary tuberculosis. Am J Respir Crit Care Med 2014; 190: 1053-1066.

129 Stenmark KR, Frid MG, Yeager ME. Fibrocytes: potential new therapeutic targets for pulmonary hypertension? Eur Respir J 2010; 36: 1232-1235.

130 Perros F, Dorfmüller P, Souza R, et al. Dendritic cell recruitmentvin lesions of human and experimental pulmonary hypertension. Eur Respir J 2007; 29: 462-468.

131 Ulrich S, Nicolls MR, Taraseviciene L, et al. Increased regulatory and decreased CD8+ cytotoxic T cells in the blood of patients with idiopathic pulmonary arterial hypertension . Respiration 2008; 75: 272-280.

132 Austin ED, Rock MT, Mosse CA, et al. T lymphocyte subset abnormalities in the blood and lung in pulmonary arterial hypertension. Respir Med 2010; 104: 454-462.

133 Yeager ME, Nguyen CM, Belchenko DD, et al. Circulating myeloid-derived suppressor cells are increased and activated in pulmonary hypertension. Chest 2012; 141: 944-952.

134 Moss RB, Hsu YP, Olds L. Cytokine dysregulation in activated cystic fibrosis (CF) peripheral lymphocytes. Clin Exp Immunol 2000; 120: 518-525.

135 Kreindler JL, Steele C, Nguyen N, et al. Vitamin D3 attenuates Th2 responses to Aspergillus fumigatus mounted by $\mathrm{CD} 4+\mathrm{T}$ cells from cystic fibrosis patients with allergic bronchopulmonary aspergillosis. J Clin Invest 2010; 120: $3242-3254$.

136 Hartl D, Griese M, Kappler M, et al. Pulmonary T(H)2 response in Pseudomonas aeruginosa-infected patients with cystic fibrosis. J Allergy Clin Immunol 2006; 117: 204-211.

137 Barton GM. A calculated response: control of inflammation by the innate immune system. J Clin Invest 2008; 118: $413-420$.

138 Soehnlein O, Lindbom L. Phagocyte partnership during the onset and resolution of inflammation. Nat Rev Immunol 2010; 10: 427-439.

139 Poe SL, Arora M, Oriss TB, et al. STAT1-regulated lung MDSC-like cells produce IL-10 and efferocytose apoptotic neutrophils with relevance in resolution of bacterial pneumonia. Mucosal Immunol 2013; 6: 189-199.

140 De Santo C, Salio M, Masri SH, et al. Invariant NKT cells reduce the immunosuppressive activity of influenza A virus-induced myeloid-derived suppressor cells in mice and humans. J Clin Invest 2008; 118: 4036-4048.

141 Zhang C, Lei GS, Shao S, et al. Accumulation of myeloid-derived suppressor cells in the lungs during Pneumocystis pneumonia. Infect Immun 2012; 80: 3634-3641.

142 Lei GS, Zhang C, Lee CH. Myeloid-derived suppressor cells impair alveolar macrophages through PD-1 receptor ligation during Pneumocystis pneumonia. Infect Immun 2015; 83: 572-582.

143 Zhang DH, Cohn L, Ray P, et al. Transcription factor GATA-3 is differentially expressed in murine Th1 and Th2 cells and controls Th2-specific expression of the interleukin-5 gene. J Biol Chem 1997; 272: 21597-21603.

144 Zheng W, Flavell RA. The transcription factor GATA-3 is necessary and sufficient for Th2 cytokine gene expression in CD4T cells. Cell 1997; 89: 587-596.

145 Zhu J, Cote-Sierra J, Guo L, et al. Stat5 activation plays a critical role in Th2 differentiation. Immunity 2003; 19: 739-748.

$146 \mathrm{Hu} \mathrm{H}$, Huston G, Duso D, et al. CD4(+) T cell effectors can become memory cells with high efficiency and without further division. Nat Immunol 2001; 2: 705-710.

147 De Wilde V, Van Rompaey N, Hill M, et al. Endotoxin-induced myeloid-derived suppressor cells inhibit alloimmune responses via heme oxygenase-1. Am J Transplant 2009; 9: 2034-2047.

148 Nefedova Y, Huang M, Kusmartsev S, et al. Hyperactivation of STAT3 is involved in abnormal differentiation of dendritic cells in cancer. J Immunol 2004; 172: 464-474.

149 Chalmin F, Ladoire S, Mignot G, et al. Membrane-associated Hsp72 from tumor-derived exosomes mediates STAT3-dependent immunosuppressive function of mouse and human myeloid-derived suppressor cells. $J$ Clin Invest 2010; 120: 457-471.

150 Serafini P, Mgebroff S, Noonan K, et al. Myeloid-derived suppressor cells promote cross-tolerance in B-cell lymphoma by expanding regulatory T cells. Cancer Res 2008; 68: 5439-5449.

151 Varga G, Ehrchen J, Tsianakas A, et al. Glucocorticoids induce an activated, anti-inflammatory monocyte subset in mice that resembles myeloid-derived suppressor cells. J Leukoc Biol 2008; 84: 644-650. 